Document downloaded from:

http://hdl.handle.net/10251/180311

This paper must be cited as:

Andrés, D.; Vappou, J.; Jimenez, N.; Camarena Femenia, F. (2021). Ultrasonic Holograms to Enhance Hyperthermia Volumes. IEEE. 1-4.

https://doi.org/10.1109/IUS52206.2021.9593807

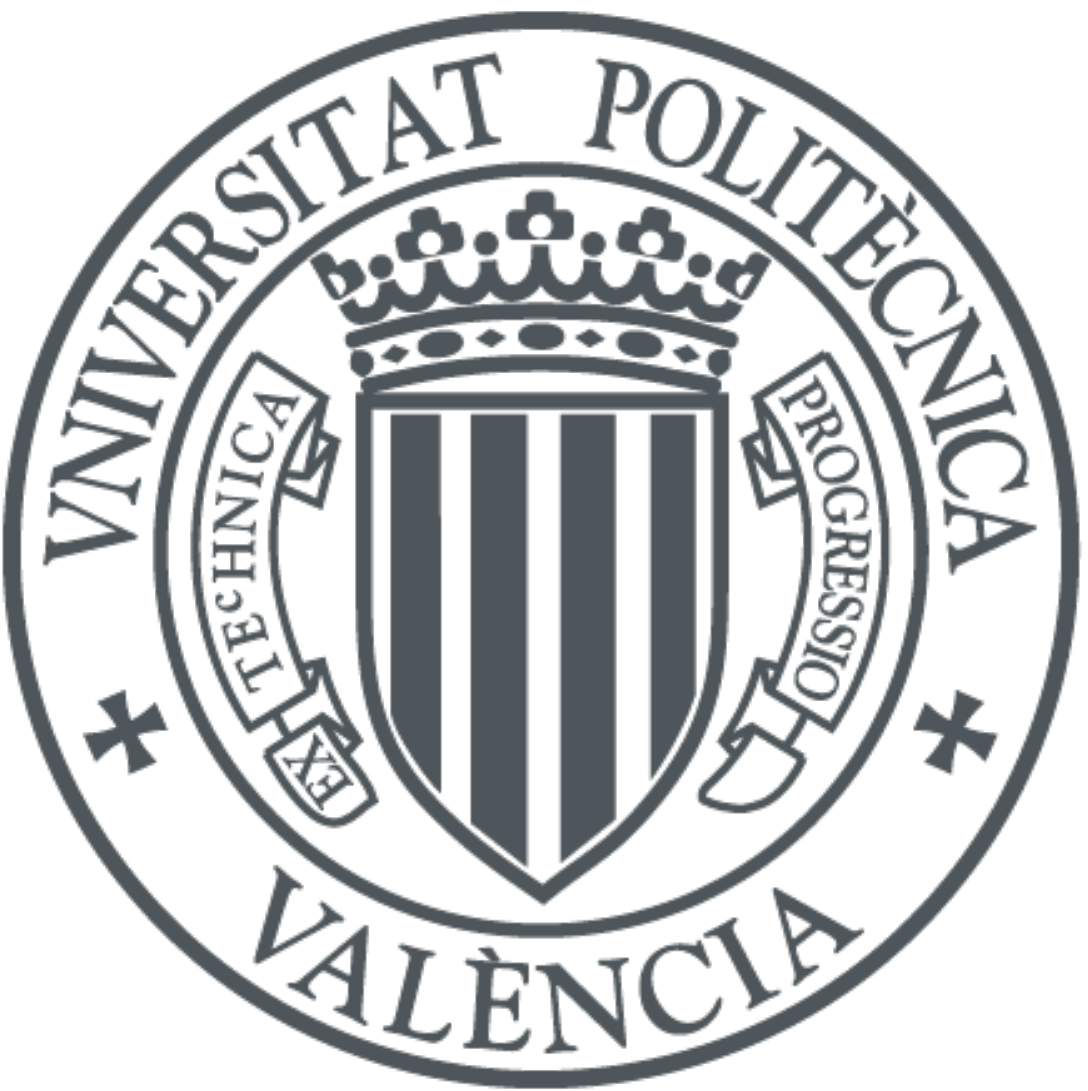

The final publication is available at

https://doi.org/10.1109/IUS52206.2021.9593807

Copyright IEEE

Additional Information 


\title{
Ultrasonic Holograms to Enhance Hyperthermia Volumes
}

\author{
Diana Andrés *, Jonathan Vappou †, Noé Jiménez *, Francisco Camarena * \\ *Instituto de Instrumentación para Imagen Molecular, Universitat Politècnica de València, Spain \\ Email: diaanbau@upv.es \\ †ICube Laboratory, CNRS-Strasbourg University, France \\ Email: jvappou@unistra.fr
}

\begin{abstract}
In this work we numerically and experimentally study the performance of large-volume hyperthermia induced by acoustic holographic-modulated high-intensity focused ultrasound (AHmHIFU) in gelatin phantoms. Excellent agreement was found between thermal simulations of the AHmHIFU system and temperature maps characterized with Proton Resonance Frequency Shift MR-thermometry at 1.5 T. ROIs inside which experimental temperature elevation was greater than 4 degrees were $11 \mathrm{~mm}$ by $16 \mathrm{~mm}$ and $7 \mathrm{~mm}$ by $30 \mathrm{~mm}$ with and without the holographic lens, respectively. Most importantly, standard deviation inside these ROIs were equal to $12 \%$ and $40 \%$ of the maximum temperature with and without the lens. This demonstrates that a wider and more uniform heated region can be generated using the holographic lens, showing the capabilities of acoustic holograms to be used as a low-cost alternative for large-volume hyperthermia treatments
\end{abstract}

Index Terms-Ultrasound, acoustic holograms, hyperthermia.

\section{INTRODUCTION}

Therapeutic ultrasound is an emerging technique that has many applications thanks to its non-invasive and non-ionizing nature. These therapies rely on the interaction between ultrasound waves and tissues, involving mechanical and/or thermal mechanisms. Indications range from low intensity applications, mainly conceived for transcranial therapies such as blood-brain barrier opening [1] or neuromodulation [2], to high intensity therapies such as histotripsy [3], thermal ablation or hyperthermia [4].

In order to obtain biological effects, ultrasound must be directed and focused to the target to be treated. To achieve this focalization, curved [5] and phased array [6] transducers are employed, usually combined with an imaging system to monitor the treatment. Even though these systems allow for a precise location of the focus, they are very expensive and complex. To overcome this drawback, acoustic holograms have been designed and tested in water [7] and in complex media [8], where their capacity to focus on a desired target while preserving the rest of the medium was demonstrated. Acoustic holograms have also been proposed as a novel and low-cost technology for transcranial therapies such as neuromodulation or blood-brain barrier opening, in which the focal aberrations introduced by the skull are corrected, allowing the ultrasound beam to fit the shape of the target ( [8] - [9]). Holograms have also been proposed to create cavitation patterns when microbubbles are added to the sonicated medium for multiple therapeutic purposes [10].

In this work, we design and develop acoustic holograms specifically for a new therapeutic application, namely, ultrasound-induced hyperthermia [4]. Hyperthermia, defined as a mild rise in tissue temperature (usually between 39 and 45 degrees), is emerging as a highly promising therapeutic approach that has been shown to activate the immune system, and/or enhance drug delivery, in particular chemotherapeutic drug administration. We present a method based on 3Dprinted acoustic holograms to create thermal holographic patterns for local and controlled hyperthermia using a singleelement ultrasonic transducer. Resulting thermal patterns are first validated numerically. Experimental thermal patterns are measured subsequently using an infra-red camera and MRI-thermometry in phantoms and ex-vivo soft tissue.

\section{Materials And Methods}

Thermal holographic patterns are produced when acoustic energy is locally transformed into heat inside an absorbing medium. Therefore, the first step is to design an acoustic hologram capable of generating a sharp acoustic image. To do so, we make use of time reversal methods by setting a series of virtual sources at the locations where we want to maximize the acoustic pressure. The field produced by these sources is back-propagated to a plane parallel to the transducer surface, known as the holographic plane. When the phaseconjugated field is forward propagated, acoustic waves focus on the virtual sources due to reciprocity and time-reversal invariance, and a diffraction-limited image is obtained. To design and numerically validate acoustic holograms we perform calculations using the Rayleigh-Sommerfeld integral and k-space pseudo-spectral simulations using the software k-Wave. We assume the transducer is located in front of a homogeneous tissue-like media (liver), with density and sound speed values of $\rho_{t}=1079 \mathrm{~kg} / \mathrm{m}^{3}$ [11] and $c_{t}=1586 \mathrm{~m} / \mathrm{s}$ [12] respectively, and we consider that acoustic attenuation follows a power-law as $\alpha_{t}=\alpha_{0} \cdot f^{\gamma}$ where $f$ 
is the ultrasound frequency, $\alpha_{0}=0.59 \mathrm{~dB} /\left(\mathrm{cm} \cdot \mathrm{MHz}^{\gamma}\right)$ [12] and $\gamma=1.1$. To implement the holographic lens in the 3D simulations we make use of the complex-conjugated wavefront at the holographic plane. This plane is divided into pixels of uniform width and different height depending on the backpropagated phase profile at the transducer working frequency. The holographic lens is designed assuming that each pixel vibrates longitudinally as a Fabry-Perot resonator and its height is obtained by a using a numerical interpolation method, as described in Ref. [9]. The values of density and sound speed of the lens were experimentally measured for Clear resin material (Formlabs, USA), being $\rho_{L}=1171 \mathrm{~kg} / \mathrm{m}^{3}, c_{L}=2580 \mathrm{~m} / \mathrm{s}$. Attenuation was fixed at $\alpha_{L}=2.72 \mathrm{~dB} /\left(\mathrm{cm} \cdot \mathrm{MHz}^{\gamma}\right)$, with $\gamma=1.1$, in agreement with previously reported values for similar photopolymers [7].

Once the acoustic hologram is designed, we calculate the corresponding thermal pattern produced in a homogeneous absorbing medium. To do so, an acoustical simulation is set using the acoustic holographic lens, and we record the maximum pressure at the stationary state in the whole medium. Then, the thermal pattern is obtained as a time-domain numerical solution of the Penne's bio-heat equation [13], given by

$$
\rho_{t} C_{t} \frac{\partial T}{\partial t}=\kappa \nabla^{2} T-W_{b} C_{b}\left(T-T_{a}\right)+Q,
$$

where $\rho_{t}$ and $C_{t}$ are the tissue density and specific heat capacity, $T$ the tissue temperature, $W_{b}$ the blood perfusion rate, $C_{b}$ the blood specific heat capacity, $T_{a}$ the blood ambient temperature, $\kappa$ the tissue thermal conductivity and $Q$ the volume rate of heat deposition, defined as

$$
Q=2 \alpha_{t} I,
$$

where $\alpha_{t}$ is the medium absorption coefficient and the intensity is given by $I=p^{2} / 2 \rho_{t} c_{t}$ where $p$ is the peak acoustic pressure at stationary state. In this work, no blood perfusion was considered $\left(W_{b}=0\right)$ to mimic the ex-vivo experimental conditions. Specific heat capacity and thermal conductivity were set to $C_{t}=3540 \mathrm{~J} /(\mathrm{kg} \cdot \mathrm{K})$ and $k=$ $0.52 \mathrm{~W} /(\mathrm{m} \cdot \mathrm{K})$, according to the values reported in the literature [11].

Thermal holograms were designed for two different transducers: a custom-designed MRI-compatible flat transducer of aperture $2 a=50 \mathrm{~mm}$ and a curved transducer with focal distance $F=100 \mathrm{~mm}$ and lateral dimensions of $132 \mathrm{~mm} \times 92 \mathrm{~mm}$, both with $1 \mathrm{MHz}$ central frequency. To perform the experimental validation, the calculated holographic lenses were 3D printed in Clear material with a Form 2 stereolithography printer (Formlabs, USA).

Two holograms were proposed for this study. First, a two-shaped image was selected, located in a plane parallel to the flat transducer surface and at $2 \mathrm{~cm}$ from its surface. This target was chosen to evaluate the possibility of generating arbitrary thermal holograms in an absorbing medium. Second, an ellipsoidal-shaped image was designed for the focused transducer. It was located at a perpendicular plane with respect to the source surface. This target was selected to show the capabilities of holograms to widen the natural focus of a focused transducer and generate large-volume thermal patterns for hyperthermia applications.

\section{A. Experimental setup}

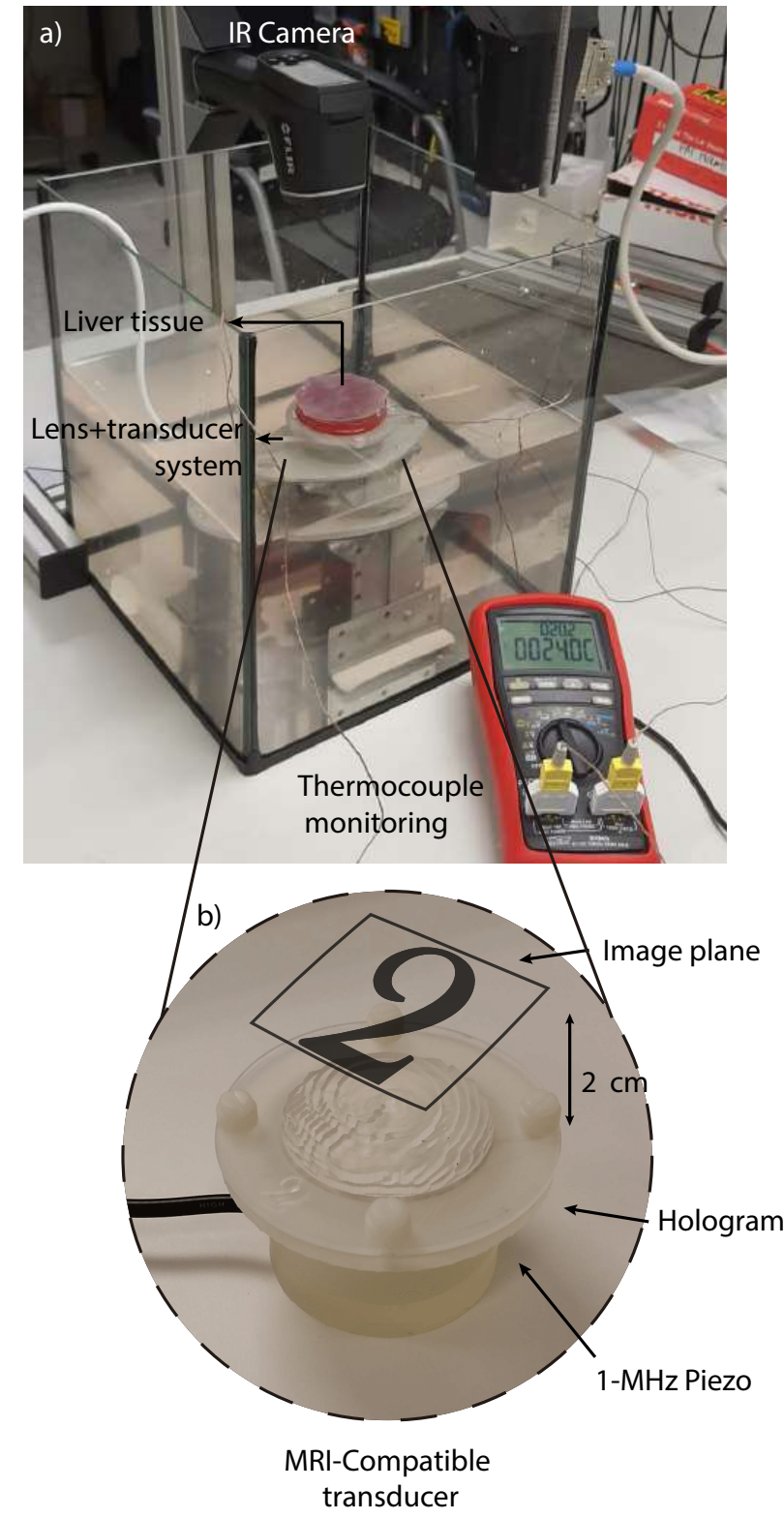

Fig. 1. ()a) Experimental setup for thermal hologram validation using an infrared thermal camera. ()b) Scheme of the employed lens-transducer system.

Acoustic measurements were performed in a degassed water tank at $23{ }^{\circ} \mathrm{C}$. Acoustic lenses were coupled to the transducer surfaces with a thin layer of vaseline, ensuring no 
bubble was trapped between both objects. The transducer-lens system was driven by a 5-cycles sinusoidal pulse burst at a frequency of $f=1.1 \mathrm{MHz}$ using a signal generator (14 bits, $100 \mathrm{MS} / \mathrm{s}$, model PXI5412, National Instruments, USA) and amplified by a linear RF amplifier (ENI 1040L, $400 \mathrm{~W}, 55 \mathrm{~dB}$, ENI, Rochester, NY, USA). The pressure field was measured with a piezoelectric hydrophone $(-225.5 \mathrm{~dB}$ re $1 \mathrm{~V} / \mu \mathrm{Pa}$ at $1 \mathrm{MHz}$, Model Y-104, Sonic Concepts, USA), calibrated from $40 \mathrm{kHz}$ to $2 \mathrm{MHz}$. The field was scanned using a 3D positioning system (10 $\mu \mathrm{m}$ precision, OWIS GmbH, Germany) from $-22 \mathrm{~mm}<\mathrm{x}<22 \mathrm{~mm}$ and $-22 \mathrm{~mm}<\mathrm{y}<22 \mathrm{~mm}$, at $\mathrm{z}=20 \mathrm{~mm}$ using steps of $1 \mathrm{~mm}$, and at each location the signals were averaged 20 times.

Thermal holograms were validated with two different systems: A thermal camera for the flat transducer, and MR Thermometry for the focused transducer. On the one hand, the evolution of the thermal pattern was observed with an infrared thermal camera (FLIR TG165, Teledyne FLIR, USA), at the surface of a $20-\mathrm{mm}$ slice of a beef liver, the experimental setup is shown in Figure 1 (a). Tissue was previously degassed using a vacuum pump. Thermal images were obtained at a rate of 11 images per minute. During thermal experiments, the single element transducer was driven with a continuous sinusoidal signal (1.1 MHz, $25 \mathrm{~W})$. In addition, two thermocouples (5SRTC, Omega Engineering, USA) were positioned at the surface of the lens and inside the surrounding water to monitor the temperature. Fig. 1 (b) shows in detail the lens-transducer system employed in the experiment for the two-shaped hologram.

In the case of the focused transducer, thermal holograms were measured using Proton Resonance Frequency Shift MRthermometry in a milk-gelatin based phantom made with $4 \%$ gelatin-30\% concentrated milk. Experiments were performed in a $1.5 \mathrm{~T}$ Aera MRI (Siemens, Erlangen, Germany). A single-slice 2D gradient-echo (GRE) sequence was used for MR PRFS Thermometry, allowing the reconstruction of one thermal image every 1.2 seconds.

\section{RESULTS}

\section{A. Two-shaped hologram}

First, the two-shaped acoustic hologram was measured in water with a spatial step of $1 \mathrm{~mm} \times 1 \mathrm{~mm}$ to compare results with the simulated acoustic field, shown in Fig. 2 (a). The experimental pressure field, depicted in Fig. 2 (b), was found to be in great agreement with the simulated one. Discrepancies between both fields might be related to the fact that the holographic lens was designed for a liver-like media and measured in water, which has slightly different properties in terms of acoustic impedance, internal tissue structures, misalignments between source and tissue, and because the axial distance between the transducer and the measurement plane might be not exactly the same as the simulated.
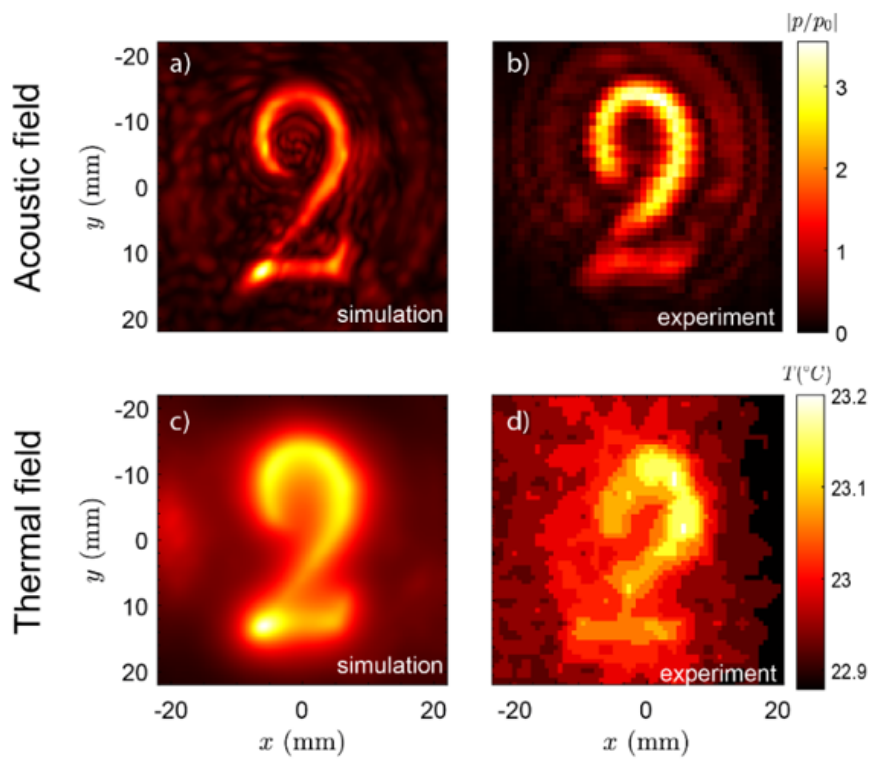

Fig. 2. (a) Simulated acoustic pressure field. (b) Acoustic pressure field measured in degassed water. (c) Simulated thermal pattern in liver tissue. (d) Thermal pattern measured with an infrared camera in liver tissue.

Second, the temperature rise was measured at the surface of the liver tissue using the infrared thermal camera. Both, simulated, Fig. 2 (c), and measured, Fig. 2 (d), thermal patterns agree at the same instant of time (1 minute) when considering continuous acoustic signal. The thermal image is well defined until heat diffusion dominates and all the central part of the hologram is heated almost uniformly, as it also occurs in simulations.

\section{B. Large volume hyperthermia hologram}

To enlarge and shift the natural focus of the focused transducer, we have designed a $1.5-\mathrm{cm}$ wide ellipsoidal hologram located at $65 \mathrm{~mm}$ from the transducer surface, while its natural focus is $1.5 \mathrm{~mm}$ wide at $10 \mathrm{~cm}$ depth. Both acoustic and thermal holograms were obtained numerically, while the hyperthermia region was evaluated experimentally using MR-thermometry in a gelatin-milk based phantom.

Excellent agreement was found between thermal simulations and temperature maps measured by MRT. ROIs inside which experimental temperature elevation was greater than 4 degrees were $15 \mathrm{~mm} \times 16 \mathrm{~mm}$ and $7 \mathrm{~mm} \times 25 \mathrm{~mm}$ with and without the holographic lens, respectively, while these same regions have $11 \mathrm{~mm} \times 16 \mathrm{~mm}$ and $7 \mathrm{~mm} \times 30 \mathrm{~mm}$ sizes in the experiment. Most importantly, standard deviation inside these regions were equal to $12 \%$ and $40 \%$ of the maximum temperature with and without the lens. This demonstrates that a wider and more uniform heating region can be achieved using the holographic lens. Moreover, focal region is steered $35 \mathrm{~mm}$ towards the transducer surface, which can be clearly seen in both simulation and experiment. Note this technology 


\section{Thermal simulation}

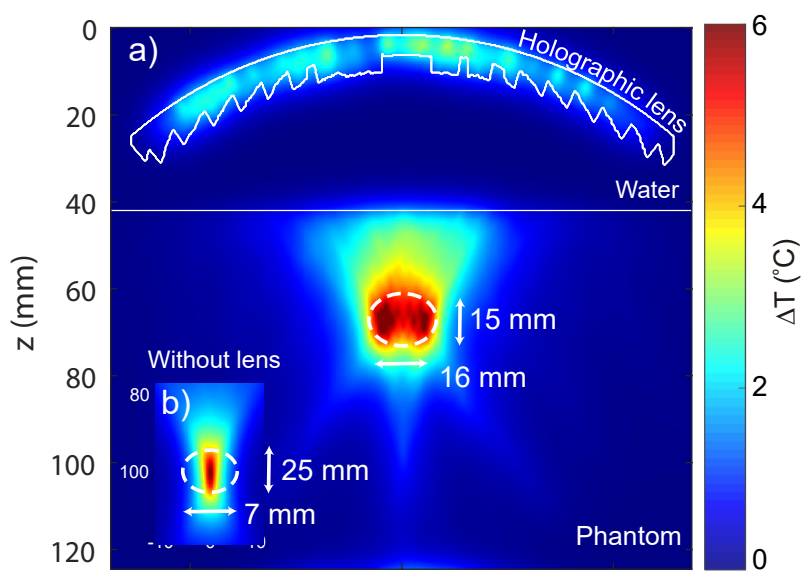

1.5-Tesla MRI thermometry

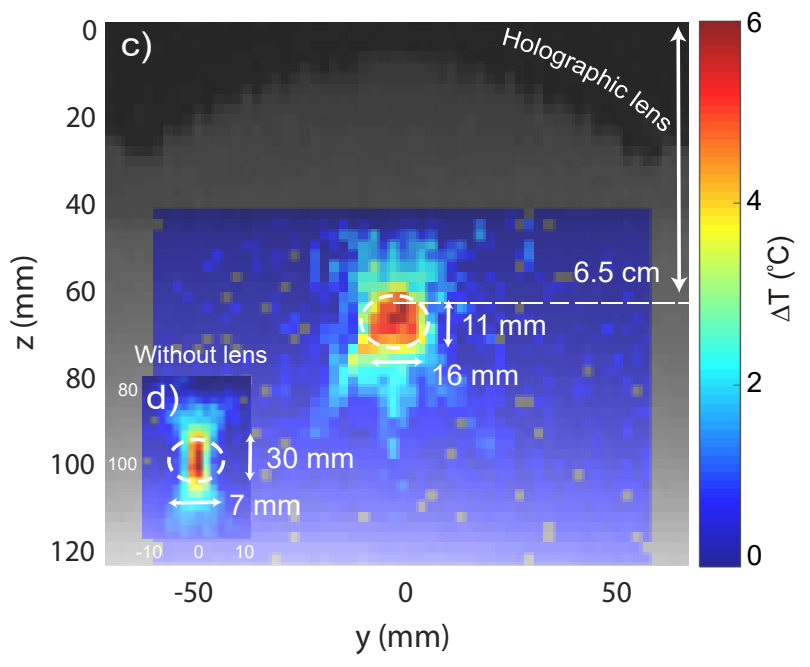

Fig. 3. (a) Thermal simulation for a 1-cm wide hologram located at $65 \mathrm{~mm}$ from the transducer surface and (b) for the focused transducer without the lens. (c) MRI-thermometry measurement for the hologram and (d) for the focused transducer without the lens.

allows not only to enlarge focus size but also to change its position.

\section{Conclusions}

In this work we have numerically calculated and experimentally validated thermal holograms in an ex-vivo tissue and tissue mimicking gelatin-milk phantom. Acoustic and thermal simulations, infrared images, and magnetic resonance thermometry measurements are in good agreement with the proposed designs for all the studied cases. These results demonstrate the capability of holographic lenses coupled to plane and focused ultrasound transducers to generate arbitraryshaped thermal patterns in absorbing media. This technology can be used to enlarge and relocate the focus of existing devices or to match the shape of the thermal hologram to complex-shaped target regions. Using a single sonication, acoustic holograms allow large volume hyperthermia treatments using a low-cost and personalized system.

\section{ACKNOWLEDGMENTS}

This research has been supported by the Spanish Ministry of Science, Innovation and Universities (MICINN) through grants IJC2018-037897-I, FPU19/00601 and PID2019-111436RB$\mathrm{C} 22$, by the Agència Valenciana de la Innovació through grants INNCON/2021/8 and INNVA1/2020/92, by Generalitat Valenciana through grant AICO/2020/268. Action co-financed by the European Union through the Programa Operativo del Fondo Europeo de Desarrollo Regional (FEDER) of the Comunitat Valenciana 2014-2020 (IDIFEDER/2018/022) and (IDIFEDER/2021/004).

\section{REFERENCES}

[1] N. McDannold, C. D. Arvanitis, N. Vykhodtseva, and M. S. Livingstone, "Temporary disruption of the blood-brain barrier by use of ultrasound and microbubbles: safety and efficacy evaluation in rhesus macaques," 2012.

[2] J. Blackmore, S. Shrivastava, J. Sallet, C. R. Butler, and R. O. Cleveland, "Ultrasound neuromodulation: a review of results, mechanisms and safety," Ultrasound in medicine \& biology, vol. 45, no. 7, pp. 15091536, 2019.

[3] W. W. Roberts, T. L. Hall, K. Ives, J. S. Wolf, J. B. Fowlkes, and C. A. Cain, "Pulsed cavitational ultrasound: a noninvasive technology for controlled tissue ablation (histotripsy) in the rabbit kidney," The Journal of urology, vol. 175, no. 2, pp. 734-738, 2006.

[4] M. D. Gray, P. C. Lyon, C. Mannaris, L. K. Folkes, M. Stratford, L. Campo, D. Y. Chung, S. Scott, M. Anderson, R. Goldin, et al., "Focused ultrasound hyperthermia for targeted drug release from thermosensitive liposomes: results from a phase i trial," Radiology, vol. 291, no. 1, pp. 232-238, 2019

[5] A. N. Pouliopoulos, S.-Y. Wu, M. T. Burgess, M. E. Karakatsani, H. A. Kamimura, and E. E. Konofagou, "A clinical system for noninvasive blood-brain barrier opening using a neuronavigation-guided single-element focused ultrasound transducer," Ultrasound in medicine \& biology, vol. 46, no. 1, pp. 73-89, 2020.

[6] A. Abrahao, Y. Meng, M. Llinas, Y. Huang, C. Hamani, T. Mainprize, I. Aubert, C. Heyn, S. E. Black, K. Hynynen, et al., "First-in-human trial of blood-brain barrier opening in amyotrophic lateral sclerosis using mr-guided focused ultrasound," Nature communications, vol. 10, no. 1, pp. 1-9, 2019

[7] K. Melde, A. G. Mark, T. Qiu, and P. Fischer, "Holograms for acoustics," Nature, vol. 537, no. 7621, pp. 518-522, 2016.

[8] G. Maimbourg, A. Houdouin, T. Deffieux, M. Tanter, and J.-F. Aubry, "3d-printed adaptive acoustic lens as a disruptive technology for transcranial ultrasound therapy using single-element transducers," Physics in Medicine \& Biology, vol. 63, no. 2, p. 025026, 2018.

[9] S. Jiménez-Gambín, N. Jiménez, J. M. Benlloch, and F. Camarena, "Holograms to focus arbitrary ultrasonic fields through the skull," Physical Review Applied, vol. 12, no. 1, p. 014016, 2019.

[10] J. Kim, S. Kasoji, P. G. Durham, and P. A. Dayton, "Acoustic holograms for directing arbitrary cavitation patterns," Applied Physics Letters, vol. 118 , no. 5, p. 051902, 2021.

[11] R. L. Mcintosh and V. Anderson, "A comprehensive tissue properties database provided for the thermal assessment of a human at rest," Biophysical Reviews and Letters, vol. 5, no. 03, pp. 129-151, 2010.

[12] B. E. Hammer, "Physical properties of tissues," Radiology, vol. 181, no. 1, pp. 128-128, 1991.

[13] H. H. Pennes, "Analysis of tissue and arterial blood temperatures in the resting human forearm," Journal of applied physiology, vol. 1, no. 2, pp. 93-122, 1948. 